\title{
Radial variation of elastic properties of SCS-6 silicon carbide fiber
}

\author{
Shamachary Sathish \\ Analytical Services and Materials, Inc., 107 Research Drive, Hampton, Virginia 23666
}

John H. Cantrell and William T. Yost

NASA Langley Research Center, Mail Stop 231, Hampton, Virginia 23681-0001

(Received 21 December 1993; accepted 26 April 1994)

\begin{abstract}
The upper and lower bounds of the bulk, shear, and Young's moduli are calculated pointby-point along the radius of SCS-6 silicon carbide fibers using the Hashin-Shtrikman equations from considerations of the Auger spectra along the fiber radius. The calculated values are in agreement with measurements of the average Young modulus obtained over relatively large radial regions of the fiber using scanning acoustic microscopy (SAM). The validity of the Hashin-Shtrikman bounds calculations is enhanced by the agreement found between experimental SAM $V(z)$ curves for fixed $z$ along the fiber radius and the theoretical bounds of such curves obtained from the bounds of the calculated bulk and shear moduli.
\end{abstract}

\section{INTRODUCTION}

Ceramic fibers are being used as reinforcement materials in both metal and ceramic matrix composites developed for high temperature applications. The high tensile strength, low mass density, low thermal expansivity, and high resistance to degradation in hostile environments have made these fibers attractive for structural applications. One of the most widely used fibers is SCS-6 silicon carbide fiber. ${ }^{1}$ The SCS-6 fibers are produced by high temperature $\left(1100-1300^{\circ} \mathrm{C}\right)$ chemical vapor deposition of a $50 \mu \mathrm{m}$ thick silicon carbide layer on a $33 \mu \mathrm{m}$ diameter carbon filament. The $\mathrm{SiC}$ layer in turn is coated with two layers of carbon for shielding. The outer layer of carbon improves the strength of the fiber and tailors the matrix-fiber interfaces for the fabrication of composites.

The microstructure of SCS- 6 fibers has been studied extensively by Ning and Pirouz ${ }^{2}$ and Ning et al. ${ }^{3}$ using transmission electron microscopy (TEM), high resolution transmission electron microscopy (HREM), and electron diffraction. According to their observations the central or core portion of the SCS-6 fiber is a filament $33 \mu \mathrm{m}$ in diameter, consisting of turbostratic carbon (TC) blocks having dimensions in the range $1-50 \mathrm{~nm}$. The $c$-axes of these blocks are randomly oriented throughout the core region. Surrounding the core region is a $1.5 \mu \mathrm{m}$ thick coating of pyrolitic carbon, consisting of TC blocks with dimensions in the range $30-50 \mathrm{~nm}$ that are arranged with the $c$-axes of the blocks preferentially (but not exclusively) aligned along the direction radial to the carbon filament axis. This inner coating of pyrolitic carbon thus has microtexture. Immediately outside the pyrolitic carbon layer are four layers of silicon carbide. The layer SiC-1 adjacent to the inner carbon coating consists of rod-like grains of $\beta-\mathrm{SiC}$. Nearest the inner carbon coating the $\beta-\mathrm{SiC}$ grains are roughly $5-15 \mathrm{~nm}$ in length and are randomly oriented. At larger distances from the inner carbon coating the grains become larger (of the order $50-150 \mathrm{~nm}$ in length) and become increasingly aligned along the fiber radial direction. Adjacent to the SiC-1 layer is a $\mathrm{SiC}-2$ layer with a pronounced twin orientation between the grains. The $\mathrm{SiC}-3$ layer adjacent to the $\mathrm{SiC}-2$ layer is a region of heavily faulted grains, with the faulting increasing as the grain size increases. The SiC-4 layer, starting at approximately $15 \mu \mathrm{m}$ from the core region, is clearly demarcated from the SiC-3 layer by the appearance of grains that are roughly twice the size of the grains in the $\mathrm{SiC}-3$ layer. At the extreme edge of the fiber is a coated layer consisting of $\mathrm{SiC}$ particles embedded in a carbon matrix (referred to as the outer carbonaceous layer).

Ning and Pirouz ${ }^{2}$ have also investigated the chemical composition across the diameter of fiber using scanning Auger microscopy, electron energy loss spectroscopy (EELS), and parallel EELS (PEELS). Their results show that the regions $\mathrm{SiC}-1, \mathrm{SiC}-2$, and SiC-3 have extra carbon atoms compared to that of the stoichiometric layer SiC-4. The concentration of carbon is highest near the carbon coating and decreases as the radial distance increases toward the stoichiometric SiC-4 layer. At each boundary between different layers of $\mathrm{SiC}$ a drop in carbon concentration is observed. The largest drop occurs between the $\mathrm{SiC}-3$ and the SiC-4 boundary. Correspondingly, jumps in silicon concentrations are observed at the boundaries. The SiC-4 
layer is nearly stoichiometric. Toward the radial edge of the fiber the carbon concentration increases and the silicon concentration decreases. The outermost fiber layer (carbonaceous layer) has been shown to consist of three sublayers. The middle sublayer has a relatively low density of $\mathrm{SiC}$ particles embedded in the carbon matrix, while the layer closest to SiC- 4 consists of a large concentration of $\mathrm{SiC}$ particles embedded in the carbon matrix. The outer carbonaceous layer is thus quite different in composition from the inner pyrolitic carbon coating.

The complex microstructure and chemical composition have considerable impact on the mechanical properties of the SCS- 6 fibers. In composites with large diameter SCS fibers, the fiber microstructure plays a dominant role in determining the ultimate strength of the composite. The purpose of the present paper is to obtain a point-by-point radial profile of the bulk and shear moduli of SCS- 6 fibers. By assuming that the carbon concentrations in the various $\mathrm{SiC}$ layers obey the law of mixtures, ${ }^{2,4}$ the volume fractions of carbon and silicon carbide in the fiber layers can then be estimated from scanning Auger microscopy. The estimated volume fractions are used in the Hashin-Shtrikman equations ${ }^{5}$ to estimate the upper and lower bounds of the bulk and shear moduli point-by-point along the fiber radius. These results are then used to calculate the upper and lower bounds of the acoustic reflectivity profile expected from scanning acoustic microscopy (SAM) over the same radial region of the fiber. The theoretical SAM reflectivity profile is compared to the experimental reflectivity profile to obtain an estimate of the validity of the elastic moduli calculations. Finally, the Hashin-Shtrikman bounds of Young's modulus is calculated over the fiber radius, and the results are compared to experimental measurements of the average Young modulus over various regions of the fiber radius.

\section{THEORETICAL EVALUATION OF ELASTIC MODULI PROFILE ALONG FIBER RADIUS}

Scanning Auger microscopy of SCS-6 fibers ${ }^{2}$ reveals that the concentration of carbon atoms decreases with increasing radial distance from the edge of the carbon core up to the outer carbonaceous coatings. The carbon concentration reaches the stoichiometric proportion in the $\mathrm{SiC}-4$ layer and then increases as the outermost carbon coatings are approached. The drop in carbon concentration near the interface of the carbon core and the SiC-1 layer is very sharp, and at each boundary between the different SiC layers a sudden, though less dramatic, drop in concentration is observed. The silicon concentration varies in a similar, but less pronounced, inverse fashion with radial distance from the carbon core. The most dramatic change in silicon concentration occurs at the SiC-3/SiC-4 boundary. Near the edge of the fiber the relative atomic concentrations of carbon and silicon separate the carbonaceous coatings into four relatively well-demarcated sublayers. It is important to note that the extra carbon atoms in the carbon-rich $\mathrm{SiC}$ regions are thought to be concentrated at the boundaries of the $\mathrm{SiC}$ grains $^{2.3}$ and may be viewed as a mixture of carbon and fine grains of $\mathrm{SiC}^{2,4}$ This distribution of extra carbon directly affects the local values of the density and elastic moduli of the fiber.

\section{A. Hashin-Shtrikman bounds of elastic moduli}

The elastic moduli of pure $\mathrm{SiC}$ are very high compared to that of pure carbon. In the carbon-rich $\mathrm{SiC}$ regions, the moduli of the mixtures are much different from that of $\mathrm{C}$ and $\mathrm{SiC}$ constituents alone. However, the theoretical evaluation of the moduli of such mixtures from the moduli of the constituents is not straightforward. Of the several theoretical methods commonly used the variational approach of the Hashin-Shtrikman ${ }^{5}$ theory is among the least stringent and most successful. The Hashin-Shtrikman model not only has been useful in predicting the upper and lower bounds of the elastic moduli of fiber reinforced composites, but also has been successful in predicting the bounds of the elastic moduli of mixtures of tungsten carbide and cobalt throughout the range of composition. ${ }^{5}$ In the WC-Co mixture cobalt is concentrated mainly at the grain boundaries and serves to bind the WC grains of the material. The analogous distribution of carbon at the boundaries of the $\mathrm{SiC}$ grains in the carbon-rich $\mathrm{SiC}$ regions of the SCS-6 fiber suggests a similar application here of the Hashin-Shtrikman model.

The Hashin-Shtrikman upper bound to the bulk modulus $K$ and shear modulus $G$ for the mixtures of carbon and silicon carbide is written in terms of the corresponding constituents' moduli as

$$
K_{u}=K_{\mathrm{SiC}}+\frac{f_{\mathrm{C}}}{\left[\frac{1}{K_{\mathrm{C}}-K_{\mathrm{SiC}}}+\frac{3 f_{\mathrm{SiC}}}{3 K_{\mathrm{SiC}}+4 G_{\mathrm{SiC}}}\right]}
$$

and

$$
\begin{aligned}
G_{u}= & G_{\mathrm{SiC}} \\
& +\frac{f_{\mathrm{C}}}{\left[\frac{1}{G_{\mathrm{C}}-G_{\mathrm{SiC}}}+\frac{6 f_{\mathrm{SiC}}\left(K_{\mathrm{SiC}}+2 G_{\mathrm{SiC}}\right)}{5 G_{\mathrm{SiC}}\left(3 K_{\mathrm{SiC}}+4 G_{\mathrm{SiC}}\right)}\right]}
\end{aligned}
$$

where $K_{\mathrm{SiC}}$ and $G_{\mathrm{SiC}}$ are, respectively, the bulk and shear moduli of stoichiometric $\mathrm{SiC}, K_{\mathrm{C}}$ and $G_{\mathrm{C}}$ are the bulk and shear moduli of carbon, and $f_{\mathrm{C}}$ and $f_{\mathrm{SiC}}$ are the volume fractions of carbon and $\mathrm{SiC}$ in the mixture. The Hashin-Shtrikman lower bounds to the moduli are obtained by interchanging the indices in Eqs. (1) and 
(2). In order to evaluate these equations at each point along the diameter of the fiber, it is necessary to know the volume fractions $f_{\mathrm{C}}$ and $f_{\mathrm{SiC}}$ of the mixture at each diametrical point of the fiber.

\section{B. Evaluation of volume fractions of mixture constituents}

The volume fractions $f_{\mathrm{C}}$ and $f_{\mathrm{SiC}}$ of mixture constituents along the radius of the SCS- 6 fiber may be estimated from consideration of the scanning Auger spectrum of the fiber cross section obtained by Ning and Pirouz. ${ }^{2}$ The signals obtained in Auger spectroscopy originate from Auger electrons emitted from a thin surface layer of the material about $1 \mathrm{~nm}$ thick, which we shall call the interaction volume $V_{0}$. The signal strength $S_{x}$ obtained in the Auger spectrum for a given atomic species $x$ is proportional to the number of atoms of that species $N_{x}$ found within the interaction volume. Let $N_{\mathrm{C}}$ and $N_{\mathrm{Si}}$ denote, respectively, the number of carbon and silicon atoms found within $V_{0}$ for stoichiometric silicon carbide. For stoichiometric composition the mass density is related to $N_{\mathrm{C}}$ and $N_{\mathrm{Si}}$ as

$$
\rho_{\mathrm{SiC}}=\frac{m_{\mathrm{C}} N_{\mathrm{C}}+m_{\mathrm{Si}} N_{\mathrm{Si}}}{V_{0}}=\frac{N\left(m_{\mathrm{C}}+m_{\mathrm{Si}}\right)}{V_{0}}
$$

where $m_{\mathrm{C}}$ and $m_{\mathrm{Si}}$ are, respectively, the atomic masses of carbon and silicon. The last equality in Eq. (3) follows from setting $N_{\mathrm{C}}=N_{\mathrm{Si}}=N$ for stoichiometric composition.

We now consider that the mass density of the $\mathrm{C}-\mathrm{SiC}$ mixture may be approximated in first order from the variations from stoichiometric composition of the numbers of carbon and silicon atoms in the interaction volume as

$$
\Delta \rho=\rho_{\mathrm{C}-\mathrm{SiC}}-\rho_{\mathrm{SiC}}=\frac{1}{V_{0}}\left(m_{\mathrm{C}} \Delta N_{\mathrm{C}}+m_{\mathrm{Si}} \Delta N_{\mathrm{Si}}\right) .
$$

The interaction volume is estimated from consideration of the Kanaya-Okayama range equation to vary insignificantly (approximately 2\%) in first approximation with respect to variations in mass density to roughly $30 \%$ and thus is assumed to be constant in obtaining Eq. (4). Since the fractional variation in Auger signal strength $\Delta S_{x} / S_{x}$ for a given atomic species is in first approximation proportional to the variation $\Delta N_{x} / N_{x}$ of the number of atoms of the species found in the interaction volume, we may write from Eq. (4)

$$
\begin{aligned}
\rho_{\mathrm{C}-\mathrm{SiC}}-\rho_{\mathrm{SiC}} & =\frac{1}{V_{0}}\left(m_{\mathrm{C}} N_{\mathrm{C}} \frac{\Delta S_{\mathrm{C}}}{S_{\mathrm{C}}}+m_{\mathrm{Si}} N_{\mathrm{Si}} \frac{\Delta S_{\mathrm{Si}}}{S_{\mathrm{Si}}}\right) \\
& =\frac{N}{V_{0}}\left(m_{\mathrm{C}} \frac{\Delta S_{\mathrm{C}}}{S_{\mathrm{C}}}+m_{\mathrm{Si}} \frac{\Delta S_{\mathrm{Si}}}{S_{\mathrm{Si}}}\right)
\end{aligned}
$$

where the last equality follows from setting $N_{\mathrm{C}}=N_{\mathrm{Si}}=$ $N$ for the originally stoichiometric composition.
Solving Eq. (3) for $N / V_{0}$ and substituting the results into Eq. (5), we obtain

$$
\begin{aligned}
\rho_{\mathrm{C}-\mathrm{SiC}}= & \rho_{\mathrm{SiC}}+\frac{\rho_{\mathrm{SiC}}}{m_{\mathrm{C}}+m_{\mathrm{Si}}} \\
& \times\left(m_{\mathrm{C}} \frac{\Delta S_{\mathrm{C}}}{S_{\mathrm{C}}}+m_{\mathrm{Si}} \frac{\Delta S_{\mathrm{Si}}}{S_{\mathrm{Si}}}\right) .
\end{aligned}
$$

A plot of the mass density of SCS-6 fiber as a function of the radial distance from the edge of the carbon core to the inner portion of the outer carbonaceous layer has been obtained from Eq. (6) using the scanning Auger spectrum reported by Ning and Pirouz. ${ }^{2}$ The results are shown in Fig. 1. The variation in the mass density is seen to be large and indicates a substantial variation in the volume fractions of mixture constituents. The volume fractions $f_{\mathrm{C}}$ and $f_{\mathrm{SiC}}$ of mixture constituents appearing in Eqs. (1) and (2) can be obtained by substituting Eq. (6) into the expressions

$$
f_{\mathrm{C}}=\frac{\rho_{\mathrm{C}-\mathrm{SiC}}-\rho_{\mathrm{SiC}}}{\rho_{\mathrm{C}}-\rho_{\mathrm{SiC}}}, \quad f_{\mathrm{SiC}}=1-f_{\mathrm{C}} .
$$

\section{Calculation of bulk and shear modull and discussion}

The calculations of the Hashin-Shtrikman bounds of elastic bulk and shear moduli are obtained from Eqs. (1), (2), (6), and (7). The calculations of the upper and lower bounds of the bulk modulus of SCS-6 fiber as a function of the radial distance from the edge of the carbon core to the inner portion of outer carbonaceous layer are plotted in Fig. 2. Corresponding plots of the shear modulus are shown in Fig. 3. In all cases a large increase in the value of the moduli occurs over the carbon-rich $\mathrm{SiC}-1$ region of the fiber. The moduli reach locally maximum values in the $\mathrm{SiC}-2$ region before decreasing. The decrease continues in the $\mathrm{SiC}-3$ region until the occurrence of a dramatic rise to the invariant moduli

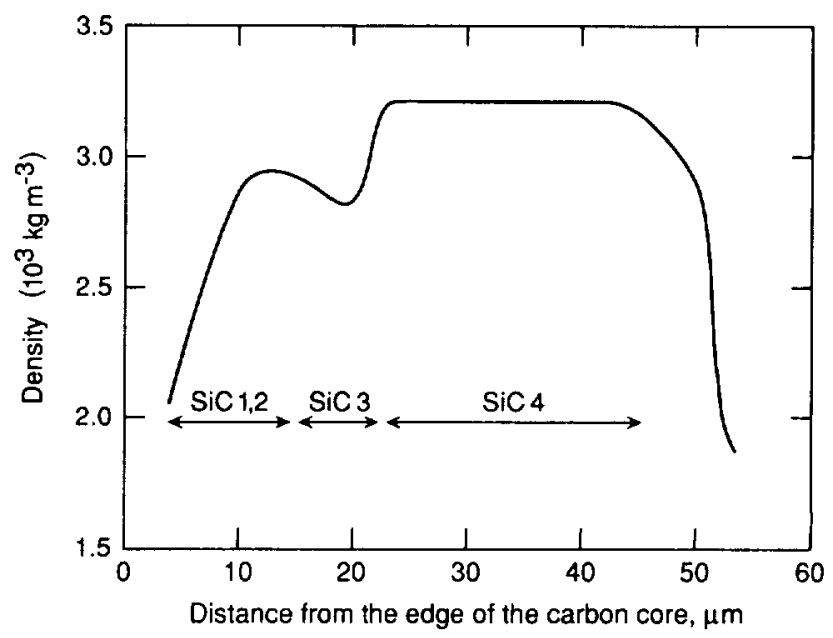

FIG. 1. Graph of variation in mass density as a function of SCS-6 fiber radius. 


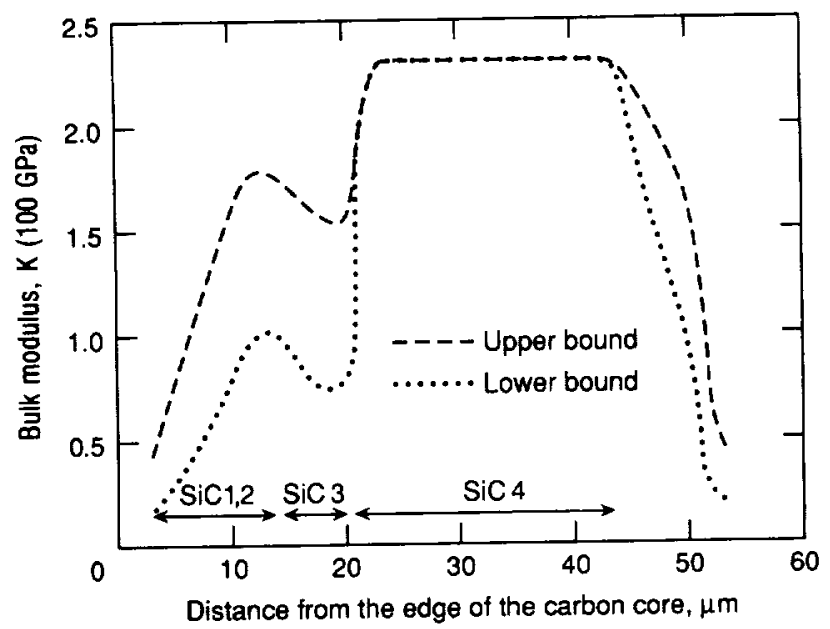

FIG. 2. Graph of Hashin-Shtrikman upper bound (dashed line) and lower bound (dotted line) of bulk modulus of SCS-6 fiber as a function of fiber radius.

values found throughout the stoichiometric SiC-4 region. The distinction between upper and lower bound values disappears in the stoichiometric $\mathrm{SiC}$ region.

\section{SCANNING ACOUSTIC MICROSCOPICAL CHARACTERIZATION OF SCS-6 FIBER CROSS SECTION}

The scanning acoustic microscope (SAM) generates images by focusing transmitted acoustic waves from a piezoelectric transducer onto a specimen surface by means of an acoustical lens. ${ }^{6}$ The acoustical coupling medium between the lens and the specimen surface is usually, but not always, water. The acoustic wave is reflected from the specimen surface and again passes through the acoustical lens prior to reception by the

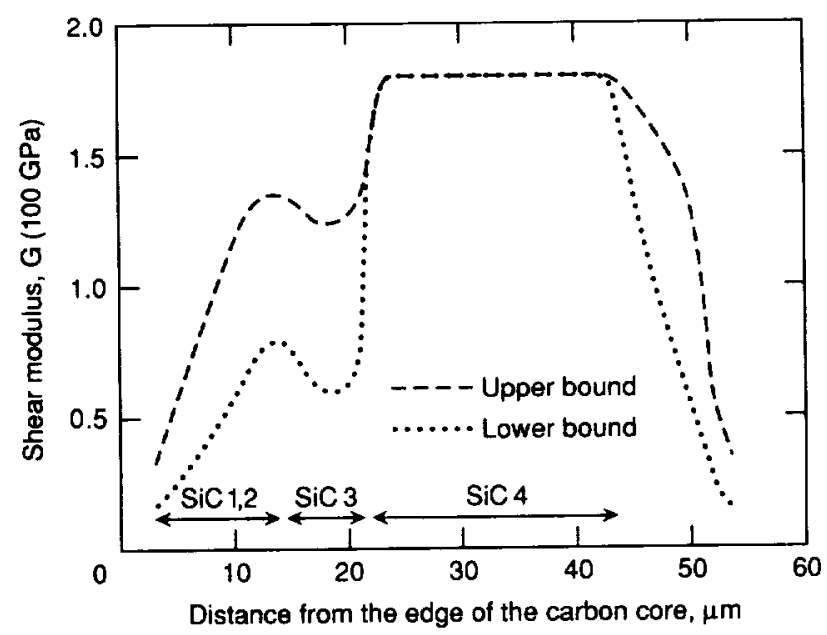

FIG. 3. Graph of Hashin-Shtrikman upper bound (dashed line) and lower bound (dotted line) of shear modulus of SCS-6 fiber as a function of fiber radius. transducer for signal processing. Images obtained when the lens is exactly focused on the surface of the specimen map the variation of the acoustic impedance (product of density and velocity of sound) of the material. Images obtained with a reduced distance between the lens and specimen (i.e., negative defocus) show an enhanced contrast when conditions are such that surface acoustic waves are generated on the specimen surface. Under such conditions the signal received by the transducer shows a periodic variation in amplitude as a function of the distance between the lens and the specimen surface. The resulting curve is known as the $V(z)$ curve or acoustic material signature (AMS). By convention the value $z=0$ corresponds to the focal plane of the lens on the specimen surface, and a decrease in the lensspecimen distance corresponds to a decrease in the value of $z$ measured from the focal plane (i.e., negative $z$ corresponds to negative defocus). An analysis of the $V(z)$ curve can yield local values of the elastic properties of the material over an area as small as $15-20 \mu \mathrm{m}^{2}$.

In the present investigation a SAM line scan across the diameter of an SCS-6 fiber with the lens-specimen distance positioned to exact focus $(z=0)$ is obtained. The experimental results are compared to a calculation of the theoretical $V(z)$ evaluated at $z=0$ for each point along the line scan in order to assess the validity of the above Hashin-Shtrikman bounds calculations.

\section{A. Theoretical $\boldsymbol{V}(\mathbf{z})$ curves}

The value of $V(z)$ where $z$ is the defocus distance measured from the focal plane of the acoustic lens is written as $^{6}$

$$
V(z)=\int_{0}^{\pi / 2} P(\theta) R(\theta) e^{-i 2 k z \cos \theta} \cos \theta \sin \theta d \theta
$$

where $\theta$ is the angle subtended with the axis of the lens at the focus, $k$ is the wave number in the coupling fluid (water was used in the experiments reported here), $P(\theta)$ is the pupil function that describes the amplitude of the wave transmitted through the lens, and $R(\theta)$ is the reflectance function for acoustic waves reflected at the liquid-solid interface. The reflectance function for a liquid-isotropic solid interface is written

$$
R(\theta)=\frac{Z_{l} \cos ^{2}\left(2 \theta_{t}\right)+Z_{t} \sin ^{2}\left(2 \theta_{t}\right)-Z_{w}}{Z_{l} \cos ^{2}\left(2 \theta_{t}\right)+Z_{t} \sin ^{2}\left(2 \theta_{t}\right)+Z_{w}}
$$

where $Z_{l}, Z_{t}$, and $Z_{w}$ are the acoustic impedances defined by

$$
Z_{l}=\frac{\rho_{s} \nu_{l}}{\cos \theta_{l}}, \quad Z_{t}=\frac{\rho_{s} v_{t}}{\cos \theta_{t}}, \quad Z_{w}=\frac{\rho_{w} \nu_{w}}{\cos \theta}
$$

and where $\rho_{w}$ and $v_{w}$ are, respectively, the mass density and the velocity of sound in water; $\rho_{s}, v_{l}$, and $v_{t}$ 
are, respectively, the mass density, longitudinal wave velocity, and shear wave velocity in the specimen; $\theta$ is the angle of incidence of acoustic waves on the specimen surface; and $\theta_{l}$ and $\theta_{t}$ are the angles of refraction of the longitudinal and shear waves in the specimen, respectively, all measured with respect to the $z$-axis.

Since the pupil function is generally constant for a given experimental setup, $V(z)$ for fixed $z$ is determined primarily by the reflectance function according to Eq. (9). The reflectance function depends on the various sound velocities via the acoustic impedances defined in Eq. (10), But the sound velocity $v_{w}=\left(B / \rho_{w}\right)^{1 / 2}$ where $B$ is the bulk modulus of water (the acoustic coupling medium) and the sound velocities $v_{l}$ and $v_{t}$ are given by $v_{l}=\left(C_{11} / \rho_{s}\right)^{1 / 2}$ and $v_{t}=\left(C_{44} / \rho_{s}\right)^{1 / 2}$ where $C_{11}$ and $C_{44}$ are the second order elastic constants of the specimen. This means that the reflectance function, hence $V(z)$ for fixed $z$, depends primarily on the localized values of the second order elastic constants and mass density of the specimen for a given acoustic coupling medium.

\section{B. Theoretical $V(0)$ line scan}

The second order elastic constants $C_{11}$ and $C_{44}$ are related to the bulk modulus $K$ and shear modulus $G$ of the specimen as $C_{11}=\left(K+\frac{4}{3} G\right)$ and $C_{44}=G$. These relations together with Eqs. (8)-(10) allow us to calculate the upper and lower bounds to $V(z)$ from our calculations of the Hashin-Shtrikman bounds of the bulk and shear moduli given in Sec. II. The calculations are simplified by setting $z=0$ in Eq. (8), corresponding to a configuration of exact focus of the lens on the specimen surface. Such calculations were performed at $1 \mu \mathrm{m}$ increments along the radius of the SCS- 6 fiber from the edge of the carbon core to the inner portion of the outer carbonaceous layer. The results of the calculations normalized to that of the carbon core are plotted in Fig. 4, where the upper bound $V(0)$ curve is given by the dashed line and the lower bound $V(0)$ curve is given by the dotted line.

\section{Experimental $V(0)$ line scans and discussion}

A Ti(15-3) metal matrix composite specimen with uniaxially embedded SCS-6 fibers was sectioned to position the axes of the fibers perpendicular to the specimen surface. The composite specimen was polished with $0.25 \mu \mathrm{m}$ diamond grit in the final stage of preparation. A high resolution scanning acoustic microscope, working at a frequency of $1.0 \mathrm{GHz}$ with the lens focused on the specimen surface $(z=0)$, was used to obtain radial line scans of the exposed fibers. A drop of distilled water was used to couple the acoustic waves from the lens to the specimen. In this mode of operation the theoretical resolution of the microscope is about $1 \mu \mathrm{m}$.

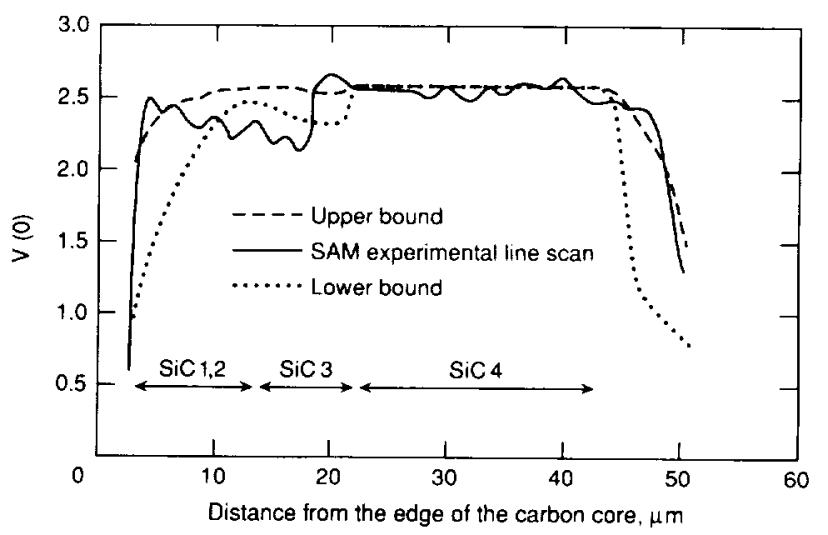

FIG. 4. Comparison of SAM experimental line scan (solid line) and SAM theoretical upper bound line scan (dashed line) and lower bound line scan (dotted line) obtained from Hashin-Shtrikman calculations. The plots are normalized to the carbon core.

A typical experimental line scan of an SCS-6 fiber radially scanned from the edge of the carbon core to the inner portion of the outer carbonaceous layer is plotted as the solid line in Fig. 4. The plot is normalized with respect to the $V(0)$ value obtained at the carbon core. The experimental curve is seen to follow the same general pattern as that of the theoretical upper and lower bound curves calculated in Sec. III. B. Exact agreement of detail between the theoretical and experimental curves is not to be expected, since the data used for the theoretical calculations are taken from the Auger spectrum of Ning and Pirouz ${ }^{2}$ and, thus, taken from a different specimen than that used to obtain the SAM experimental curve. Indeed, a comparison of the theoretical and experimental curves indicates possible differences in the material composition, relative sizes of regions, and diameter of the fibers used in the two studies. Nonetheless, the essential agreement between the theoretical and experimental $V(0)$ curves supports the validity of the present calculations of the upper and lower bounds of the bulk and shear moduli.

\section{CONCLUSION}

The calculations of the Hashin-Shtrikman bounds of the bulk and shear moduli, as a function of the radial distance from the carbon core to the inner portion of the outer carbonaceous region of SCS-6 fibers, indicate that a considerable variation in these moduli occurs over this region. The calculations are based on the theoretical assessment of the radial dependence of the mass density of the fiber obtained from scanning Auger spectra described in Sec. II. B. The mass density calculation together with the Hashin-Shtrikman bounds of the elastic moduli provides a theoretical assessment of the SAM $V(0)$ curve. The agreement between the theoretical and experimental $V(0)$ curve provides considerable evidence of the correctness of our calculations. 
As a final measure of the validity of the present calculations, we have calculated the Hashin-Shtrikman bounds of Young's modulus from the Hashin-Shtrikman bounds of the bulk and shear moduli. The results are given in Fig. 5 where we have plotted the upper and lower bounds as a function of the radial distance from the carbon core to the inner portion of the outer carbonaceous region of the fiber. As expected, we observed a large variation of Young's modulus over the region shown similar to that observed for the bulk and shear moduli.

These results are to be compared to a study recently performed by Sathish et al. ${ }^{7}$ on the direct assessment of the average Young modulus over various regions of SCS-6 fibers using scanning acoustic microscopy.

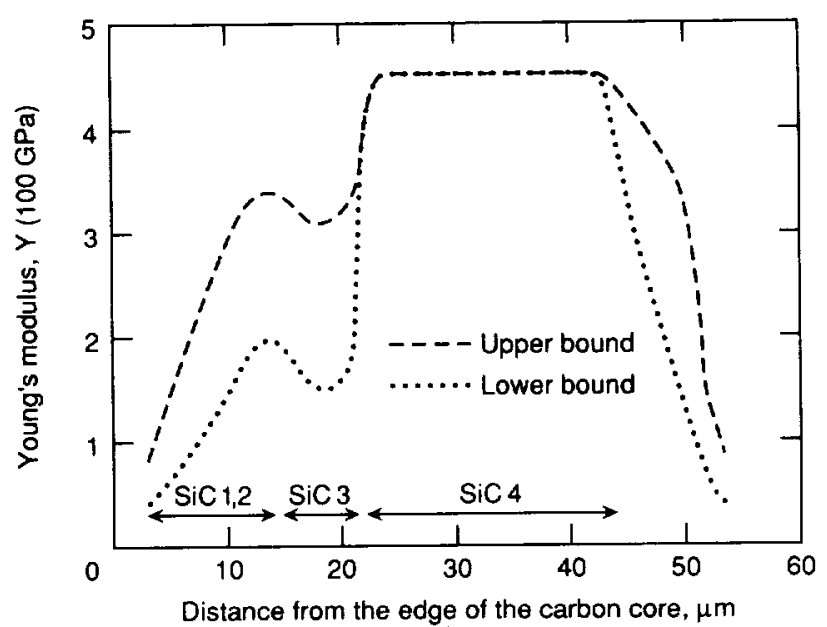

FIG. 5. Graph of Hashin-Shtrikman upper bound (dashed line) and lower bound (dotted line) of Young's modulus of SCS-6 fiber as a function of fiber radius.
The variation in image contrast obtained in the SAM micrographs was interpreted on the basis of the variation of acoustic properties in different parts of the fiber. They estimated the average Young modulus of various radially symmetric regions of the fiber from an analysis of the standing Rayleigh wave patterns developed in the regions. Their results yield an average Young modulus in the range 105-109 $\mathrm{GPa}$ for the $\mathrm{SiC}-1, \mathrm{SiC}-2$, and $\mathrm{SiC}-3$ regions and an average Young modulus of $427 \mathrm{GPa}$ for the $\mathrm{SiC}-4$ region. These results are consistent with the variations in Young's modulus observed in the graph of Fig. 5 and provide further evidence of the validity of the present calculations of the Hashin-Shtrikman bounds of the bulk and shear moduli.

\section{ACKNOWLEDGMENTS}

The authors thank Dr. E. R. Generazio and Dr. R. A. MacKay, of NASA Lewis Research Center, Cleveland, Ohio, for useful discussions and for providing the specimens used in the scanning acoustic microscopy experiments.

\section{REFERENCES}

1. Manufactured by Textron Specialty Materials, Textron Co., Inc., Lowell, MA.

2. X. J. Ning and P. Pirouz, J. Mater. Res. 6, 2234 (1991).

3. X. J. Ning, P. Pirouz, K. P. D. Lagerlof, and J. DiCarlo, J. Mater. Res. 5, 2865 (1990).

4. P. Pirouz (private communication).

5. Z. Hashin and S. Shtrikman, J. Mech. Phys. Solids 11, 1127 (1963).

6. A. Briggs, Acoustic Microscopy (Oxford University Press, 1992).

7. S. Sathish, W. T. Yost, J.H. Cantrell, E. R. Generazio, R. A. MacKay, and K. M. B. Taminger, Fourth International Symposium on Composite Materials, Cocoa Beach, FL (1993). 

